\title{
Output strictly negative imaginary systems and its connections to dissipativity theory
}

DOI:

10.1109/CDC40024.2019.9030016

\section{Document Version}

Accepted author manuscript

Link to publication record in Manchester Research Explorer

\section{Citation for published version (APA):}

Bhowmick, P., \& Lanzon, A. (2020). Output strictly negative imaginary systems and its connections to dissipativity theory. In 58th IEEE Conference on Decision and Control (pp. 6754-6759). [9030016] (Proceedings of the IEEE Conference on Decision and Control; Vol. 2019-December). IEEE.

https://doi.org/10.1109/CDC40024.2019.9030016

\section{Published in:}

58th IEEE Conference on Decision and Control

\section{Citing this paper}

Please note that where the full-text provided on Manchester Research Explorer is the Author Accepted Manuscript or Proof version this may differ from the final Published version. If citing, it is advised that you check and use the publisher's definitive version.

\section{General rights}

Copyright and moral rights for the publications made accessible in the Research Explorer are retained by the authors and/or other copyright owners and it is a condition of accessing publications that users recognise and abide by the legal requirements associated with these rights.

\section{Takedown policy}

If you believe that this document breaches copyright please refer to the University of Manchester's Takedown Procedures [http://man.ac.uk/04Y6Bo] or contact uml.scholarlycommunications@manchester.ac.uk providing relevant details, so we can investigate your claim.

\section{OPEN ACCESS}




\title{
Output strictly negative imaginary systems and its connections to dissipativity theory
}

\author{
Parijat Bhowmick and Alexander Lanzon
}

\begin{abstract}
This paper generalises the notion of output strictly negative imaginary systems and provides a complete characterisation both in frequency domain and time domain. The paper also reveals the missing link between the negative imaginary theory and dissipativity. A new time domain supply rate is introduced to characterise the class of output strictly negative imaginary systems that consists of input to the system, the derivative of an auxiliary output of the system and a real parameter $\delta>0$. Further, in addition to the output strictly negative imaginary systems, all stable negative imaginary systems are shown to be dissipative with respect to the same supply rate with $\delta=0$. An equivalence is also established between the output strictly negative imaginary systems property and time domain dissipativity of this class of systems with respect to the proposed supply rate and a specific positive definite storage function. Several numerical examples are studied to elucidate the essence of the theoretical developments.
\end{abstract}

\section{INTRODUCTION}

Negative imaginary (NI) systems theory has drawn attention from both control theorists and practising engineers over the last decade due to its wide applicability in problems such as vibration control of lightly damped flexible structures [1], cantilever beams [4], large space structures [10], robotic manipulators [10], control of nano-positioning systems [11], control of large vehicle platoons [5], observer-based control of non-square plants [15], etc. Negative imaginary systems theory was first introduced in [1] inspired by the "positive position feedback control' [6] of highly resonant mechanical systems. The negative imaginary framework offers a simple internal stability criteria $\lambda_{\max }[M(0) N(0)]<1$ for a positive feedback interconnection of two NI systems $M(s)$ and $N(s)$ of which one must be strictly negative imaginary (SNI) [1], [2]. Since the SNI system $N(s)$ must satisfy the strict frequency domain condition $j\left[N(j \omega)-N(j \omega)^{*}\right]>0$ for all $\omega \in(0, \infty)$, the existing stability results of NI-SNI interconnection fail to capture the cases where both the imaginaryHermitian parts $\left[M(s)-M(s)^{*}\right]$ and $\left[N(s)-N(s)^{*}\right]$ have transmission/blocking zeros on the $j \omega$ axis for $\omega \in(0, \infty)$. Furthermore, due to this strict frequency domain condition defined on the punctured $j \omega$ axis (i.e., excluding $\omega=0$ ), NI theory faces significant technical difficulties in both analysis and synthesis involving SNI systems. In order to circumvent these issues, a new class of strict negative imaginary systems,

This work was supported by the Engineering and Physical Sciences Research Council (EPSRC) [grant number EP/R008876/1]. All research data supporting this publication are directly available within this publication.

The authors are with the Control Systems Centre, Department of Electrical and Electronic Engineering, School of Engineering, University of Manchester, Manchester M13 9PL, U.K. Alexander.Lanzon@manchester.ac.uk (A. Lanzon) Parijat.Bhowmick@manchester.ac.uk (P. Bhowmick) termed as the Output Strictly Negative Imaginary (OSNI) systems class, has been introduced in [14] for which the strictness property is defined in terms of Output Strictly Passive (OSP) systems. An OSNI system is not required to satisfy the same strict frequency domain condition of SNI systems and hence, allows blocking/transmission zeros on the $j \omega$ axis for $\omega \in(0, \infty)$. In this paper, the notion of Output Strictly Negative Imaginary (OSNI) systems has been generalised to capture an wider class of systems. In contrast to [14], the present definition of OSNI systems (say $M(s)$ ) does not impose the full normal rank constraint on $\left[M(s)-M(s)^{*}\right]$, which implies that the OSNI systems defined in [14] is a subset of the OSNI class proposed in this paper. It is found that the OSNI and SNI subclasses intersect and the intersection contains the set of strongly strict negative imaginary (SSNI [3]) systems as illustrated via the Venn diagram (Fig. 1b). Apart from providing the definition and characterisation for OSNI systems, this paper also explores the connections between negative imaginary theory and classical dissipativity [16].

The connections between negative imaginary theory and classical dissipativity are not yet well explored. In the case of passive systems, a complete characterisation already exists in the literature using Willems's dissipative framework [16] as well as Hill-Moylan's $(Q, S, R)$-dissipative framework [17], [18]. In [24], the authors introduced the notion of 'mixed' input-output passive and finite-gain system properties using a frequency domain dissipative approach and being inspired by [24], in [8], a frequency domain $(Q, S, R)$-dissipative supply rate was introduced to characterise the class of systems with 'mixed' finite-gain and input-output (strictly) negative imaginary properties. Later on, [9] and [12] have pursued a similar approach alike [8] to establish internal stability conditions for interconnected systems with 'mixed' NI, passive and finite-gain properties. Unlike [8], [9] and [12], this paper theoretically proves that the OSNI systems with reachable (from the origin) state-space are dissipative with respect to a particular time domain supply rate $w(u, \dot{\bar{y}})=$ $2 \dot{\bar{y}}^{T} u-\delta \dot{\bar{y}}^{T} \dot{\bar{y}}$ with $\delta>0$ by showing the existence of a positive semidefinite storage function $V(x)$. Note that the auxiliary output $\bar{y}=y-D u$ is considered particularly to capture bi-proper OSNI systems; in strictly-proper cases the supply rate simplifies to $2 \dot{y}^{T} u-\delta \dot{y}^{T} \dot{y}$. This supply rate finds an interesting physical interpretation for electrical networks having voltage and charge flow as the input-output variables and for mechanical systems having force and displacement as the input-output pair. For example, in case of a springmass-damper system (Fig. 1a) being strictly-proper, the term 
$\dot{y}^{T} u$ gives the mechanical power input (velocity $(\dot{y}) \times$ force (u)) while the term $\dot{y}^{T} \dot{y}$ represents the power dissipated in the damper ( $d \dot{y}^{2}$ assuming $d=1$ ). But, for more general systems, the supply rate provides an abstraction of the net power inflow to the system and often, it is not possible to find an exact physical interpretation. It is also shown in this paper that, in addition to the OSNI systems, all stable NI systems are dissipative with respect to the same time domain supply rate $w(u, \dot{\bar{y}})$ when $\delta=0$.

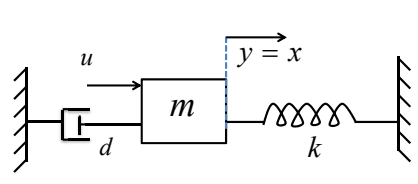

(a)

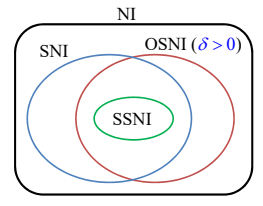

(b)
Fig. 1: (a) A spring-mass-damper system; (b) Relationship among the strict subclasses of the NI systems class.

\section{PRELIMINARIES}

In this section, some valuable technical preliminaries, definitions and lemmas are presented which underpin the proofs of the main results of the paper.

The finite dimensional, causal, LTI systems studied in this paper are described by the equations ${ }^{1}$

$$
M:\left\{\begin{array}{l}
\dot{x}=A x+B u, \quad x_{0}=x(0) ; \\
y=C x+D u,
\end{array}\right.
$$

where the input $u(t) \in \mathbb{R}^{m}$, the state $x(t) \in \mathbb{R}^{n}$ and the output $y(t) \in \mathbb{R}^{p}$ for all $t \in \mathbb{R}_{\geq 0}$. The admissible inputs are taken to be $\mathbb{R}^{m}$-valued locally square integrable functions of time $t \in \mathbb{R}_{\geq 0}$, that is, $\int_{0}^{T} u(t)^{T} u(t) \mathrm{d} t<\infty$ for all $T \in$ $[0, \infty)$. Note that the state trajectory $x(t)$ and the output $y(t)$ will also be real-valued locally square integrable functions of time $t \in \mathbb{R}_{\geq 0}$. Let us introduce the state transition function $\Phi$, associated with $M$, being a mapping from $\mathbb{R}_{\geq 0} \times \mathbb{R}_{\geq 0} \times$ $\mathbb{R}^{n} \times \mathbb{R}^{m}$ into $\mathbb{R}^{n}$. Here, $\Phi\left(t_{1}, 0, x(0), u\right)$ denotes the state $x\left(t_{1}\right)$ at time $t_{1}$ when the system $M$ starts from an initial state $x(0) \in \mathbb{R}^{n}$ at time $t=0$ and an admissible input $u(t)$ is applied on $M$ for all $t \in\left[0, t_{1}\right]$.

\section{A. Dissipative systems notations and definitions}

Let us recall the notion of dissipativity of finite dimensional, causal, LTI systems introduced in [16].

Definition 1: (Dissipative systems) [16] A dynamical system $M$, given in (1), is said to be dissipative with respect to an energy supply rate $w(u, y)$ if there exists a function $V: \mathbb{R}^{n} \rightarrow \mathbb{R}_{\geq 0}$, called the storage function, such that

$$
V(x(0))+\int_{0}^{T} w(u, y) \mathrm{d} t \geq V(x(T))
$$

for any $T \in[0, \infty)$, any initial condition $x(0) \in \mathbb{R}^{n}$ and any admissible input $u(t) \in \mathbb{R}^{m}$ for all $t \in \mathbb{R}_{\geq 0}$ where

\footnotetext{
${ }^{1}$ For simplicity of presentation, the dependence on time $t \in \mathbb{R}_{\geq 0}$ is omitted.
}

$x(T)=\Phi(T, 0, x(0), u)$ and $w(u, y)$ have been evaluated along any trajectory of (1).

Inequality (2) is known as the 'dissipation inequality' in the sense of Willems. If $V: \mathbb{R}^{n} \rightarrow \mathbb{R}_{\geq 0}$ is a differentiable storage function, then the dissipation inequality (2) can be expressed in the differential form as

$$
w(u, y) \geq \dot{V}(x) .
$$

Note that for finite dimensional LTI systems with minimal state-space realisation, the storage function $V(x)$ can be characterized with a quadratic form $x^{T} P x$, without loss of generality, where $P=P^{T}>0$ [16], [22]. Moreover, in the LTI setting, the storage function $V(x)$ can always be assumed to be a differentiable function of $x \in \mathbb{R}^{n}$ [17], [26].

For a dissipative system with a reachable (from the origin) state-space $^{2}$, the 'required supply' is defined as [21]

$$
V_{r}\left(x_{1}\right)=\inf _{\substack{x^{*} \rightarrow x_{1} \\ u(\cdot), T \leq 0}} \int_{T}^{0} w(u, y) \mathrm{d} t
$$

where $x^{*} \in \mathbb{R}^{n}$ represents the point of minimum storage. In general, the origin of a state-space is the point of minimum storage where $V\left(x^{*}\right)=V(0)=0$. The 'required supply' is the least amount of energy required to excite a system to a desired state from the state of minimum energy level [19]. $V_{r}(x)$ is a possible storage function for any dissipative system with a reachable (from the origin) state-space.

Definition 2: ( $(Q, S, R)$-dissipativity in Hill-Moylan's framework) [17] A dynamical system $M$, given in (1) with $x_{0}=0$, is said to be $(Q, S, R)$-dissipative if there exist $Q=Q^{T} \in \mathbb{R}^{p \times p}, S \in \mathbb{R}^{p \times m}$ and $R=R^{T} \in \mathbb{R}^{m \times m}$ such that

$$
\int_{0}^{T} y^{T} Q y+2 y^{T} S u+u^{T} R u \mathrm{~d} t \geq 0
$$

for all $T \in[0, \infty)$ and all admissible $u(t) \in \mathbb{R}^{m}$ for all $t \in \mathbb{R}_{\geq 0}$.

If the supply rate function in Willems's framework is specialized as $w(u, y)=y^{T} Q y+2 y^{T} S u+u^{T} R u$ where $Q=Q^{T} \in \mathbb{R}^{p \times p}, S \in \mathbb{R}^{p \times m}$ and $R=R^{T} \in \mathbb{R}^{m \times m}$, then (3) takes the form

$$
y^{T} Q y+2 y^{T} S u+u^{T} R u \geq \dot{V}(x) .
$$

\section{B. Definitions for negative imaginary systems theory}

In this subsection, we recall the definitions of NI and SNI systems.

Definition 3: (NI System) [10], [13] Let $M(s)$ be the real, rational, proper transfer function matrix of a square and causal system without any poles on the open right-half plane. $M(s)$ is said to be Negative Imaginary (NI) if

- $j\left[M(j \omega)-M(j \omega)^{*}\right] \geq 0$ for all $\omega \in(0, \infty)$ except the values of $\omega$ where $j \omega$ is a pole of $M(s)$;

\footnotetext{
${ }^{2}$ For LTI systems, reachability (to and from the origin) is equivalent to complete state controllability [20].
} 
- If $s=j \omega_{0}$ with $\omega_{0} \in(0, \infty)$ is a pole of $M(s)$, then it is at most a simple pole and the residue matrix $K_{0}=\lim _{s \rightarrow j \omega_{0}} j\left(s-j \omega_{0}\right) M(s)$ is Hermitian and positive semidefinite;

- If $s=0$ is a pole of $M(s)$, then $\lim _{s \rightarrow 0} s^{k} M(s)=0$ for all $k \geq 3$ and $\lim _{s \rightarrow 0} s^{2} M(s)$ is positive semidefinite Hermitian.

Definition 4: (SNI System) [1] Let $M(s)$ be the real, rational, proper transfer function matrix of a square and causal system. $M(s)$ is said to be Strictly Negative Imaginary (SNI) if $M(s)$ has no poles in $\Re[s] \geq 0$ and $j[M(j \omega)-$ $\left.M(j \omega)^{*}\right]>0$ for all $\omega \in(0, \infty)$.

\section{OUTPUT STRICTLY NEGATIVE IMAGINARY SYSTEMS}

In this section, we define output strictly negative imaginary (OSNI) systems ${ }^{3}$ in the frequency domain, discuss its properties and depict the set-theoretic relationship between OSNI and SNI subclasses. A state-space characterisation is also provided to test the OSNI property of an LTI system based on its minimal state-space realisation.

Definition 5: (OSNI systems) Let $M(s) \in \mathscr{R} \mathscr{H}_{\infty}^{m \times m}$. Then, $M(s)$ is said to be Output Strictly Negative Imaginary (OSNI) if there exists a scalar $\delta>0$ such that

$$
j \omega\left[M(j \omega)-M(j \omega)^{*}\right]-\delta \omega^{2} \bar{M}(j \omega)^{*} \bar{M}(j \omega) \geq 0
$$

$\forall \omega \in \mathbb{R} \cup\{\infty\}$ where $\bar{M}(j \omega)=M(j \omega)-M(\infty)$.

The parameter $\delta>0$ is an index which quantifies the level of output strictness of a given OSNI system.

Remark 1: The index $\delta=0$ corresponds to all stable NI systems because when $\delta=0$, the inequality (7) reduces simply to the negative imaginary condition $j \omega[M(j \omega)-$ $\left.M(j \omega)^{*}\right] \geq 0$ for all $\omega \in \mathbb{R} \cup\{\infty\} \Leftrightarrow j\left[M(j \omega)-M(j \omega)^{*}\right] \geq$ 0 for all $\omega \in(0, \infty)$ since the latter implies $M(0)=M(0)^{T}$ and $M(\infty)=M(\infty)^{T}$. From inequality (7), it is evident that the OSNI class is a subset of the stable NI class since (7) with $\delta>0$ implies (7) with $\delta=0$.

Example 1: Consider the SNI transfer function $N(s)=$ $s+4$ $\frac{s+4}{s^{2}+8 s+32}$. In this case, $j \omega\left[N(j \omega)-N(j \omega)^{*}\right]-\delta \omega^{2}$ $\bar{N}(j \omega)^{*} \bar{N}(j \omega)=\frac{(2-\delta) \omega^{4}-16 \delta \omega^{2}}{\left(32-\omega^{2}\right)^{2}+64 \omega^{2}} \geq 0$ for all $\omega \in$ $\mathbb{R} \cup\{\infty\}$ only when $\delta=0$. Therefore, $N(s)$ is not an OSNI (with $\delta>0$ ) system.

The above example shows that not all SNI systems are OSNI. Furthermore, in contrast to [14], the definition for OSNI systems in this paper does not explicitly invoke the OSP property and moreover, the full normal rank constraint on $\left[M(s)-M(s)^{*}\right]$ has been removed. The following example illustrates this fact.

Example 2: Let $M(s)=\frac{1}{s+1}\left[\begin{array}{ll}1 & 1 \\ 1 & 1\end{array}\right]$ which violates the full normal rank of $\left[M(s)-M(s)^{*}\right]$. To test whether $M(s)$ is OSNI or not, we apply the point-wise frequency domain condition given in Definition 5 to obtain $j \omega[M(j \omega)-$

\footnotetext{
${ }^{3}$ OSNI property is always defined for finite dimensional, causal and asymptotically stable NI systems.
}

$\left.M(j \omega)^{*}\right]-\delta \omega^{2} \bar{M}(j \omega)^{*} \bar{M}(j \omega)=\frac{(2-\delta) \omega^{2}}{1+\omega^{2}}\left[\begin{array}{ll}1 & 1 \\ 1 & 1\end{array}\right] \geq 0$ for all $\omega \in \mathbb{R} \cup\{\infty\}$ and all $\delta \in[0,2]$. Hence, it can be concluded that the system $M(s)$ is an OSNI system.

There are many SNI systems which are also OSNI systems, e.g., the SNI system $N(s)=\frac{25}{s^{2}+5 s+25}$ is also an OSNI system with $\delta \in[0,0.4]$. There however exist also some OSNI systems which are not SNI. In the SISO setting, the OSNI systems that contain zeros on the $j \omega$ axis for $\omega \in$ $(0, \infty)$ (see Example 3) are not SNI.

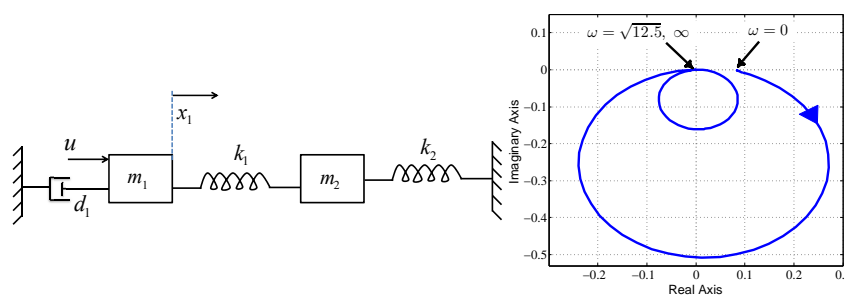

(a)

(b)

Fig. 2: (a) A mechanical system realization of an OSNI system which is not SNI; (b) Nyquist plot of the system transfer function $M(s)$ from $U(s)$ to $X_{1}(s)$.

Example 3: Consider the spring-mass-damper system shown in Fig. 2a. For the set of parameters $m_{1}=1 \mathrm{~kg}$, $m_{2}=4 \mathrm{~kg}, \quad k_{1}=30 \mathrm{~N} / \mathrm{m}, k_{2}=20 \mathrm{~N} / \mathrm{m}$ and $d_{1}=$ $1 \mathrm{Ns} / \mathrm{m}$, the above system has the transfer function $M(s)=$ $\frac{X_{1}(s)}{U(s)}=\frac{s^{2}+12.5}{s^{4}+s^{3}+42.5 s^{2}+12.5 s+150}$. It can be readily verified that $M(s)$ is an OSNI system with $\delta \in$ $[0,2]$ since $j \omega\left[M(j \omega)-M(j \omega)^{*}\right]-\delta \omega^{2} \bar{M}(j \omega)^{*} \bar{M}(j \omega)=$ $\omega^{2}(2-\delta)\left(12.5-\omega^{2}\right)^{2}$

$\overline{\left(\omega^{4}-42.5 \omega^{2}+150\right)^{2}+\omega^{2}\left(12.5-\omega^{2}\right)^{2}} \geq 0$ for all $\omega \in$ $\mathbb{R} \cup\{\infty\}$ and for all $\delta \in[0,2]$. However, this system is not SNI since $j\left[M(j \omega)-M(j \omega)^{*}\right]=0$ at $\omega=\sqrt{12.5} \mathrm{rad} / \mathrm{s}$ (see the Nyquist plot in Fig. 2b).

Based on these examples, it can be inferred that the sets of SNI and OSNI systems intersect as depicted via the Venn diagram in Fig. 1b but are not identical sets. The following lemma is a pre-requisite to prove the OSNI Lemma given in the sequel.

Lemma 1: Let $M(s) \in \mathscr{R} \mathscr{H}_{\infty}^{m \times m}$ and $\bar{M}(s)=M(s)-$ $M(\infty)$. Then, the following statements hold:

a) $M(s)$ is OSNI with $\delta>0$ if and only if $F(s)=s \bar{M}(s)$ is $\mathrm{OSP}^{4}$ and $M(\infty)=M(\infty)^{T}$;

b) $M(s)$ is NI with $\delta=0$ if and only if $F(s)=s \bar{M}(s)$ is passive and $M(\infty)=M(\infty)^{T}$.

Proof. Since $F(j \omega)+F(j \omega)^{*}-\delta F(j \omega)^{*} F(j \omega)=$ $(j \omega \bar{M}(j \omega))+(j \omega \bar{M}(j \omega))^{*}-\delta(j \omega \bar{M}(j \omega))^{*}(j \omega \bar{M}(j \omega))=$ $j \omega\left[M(j \omega)-M(j \omega)^{*}\right]-\delta \omega^{2} \bar{M}(j \omega)^{*} \bar{M}(j \omega)$ for all $\omega \in$ $\mathbb{R} \cup\{\infty\}$ by following [14, MIMO OSP Lemma] and on exploiting the property $M(\infty)=M(\infty)^{T}$, Part $a$ ) holds for $\delta>0$, while Part $b$ ) holds for $\delta=0$.

\footnotetext{
${ }^{4}$ A system $F(s) \in \mathscr{R} \mathscr{H}_{\infty}^{m \times m}$ with $F(s)+F(s)^{*}$ having full normal rank is Output Strictly Passive (OSP) if and only if there exists $\delta_{p}>0$ such that $F(j \omega)+F(j \omega)^{*} \geq \delta_{p} F(j \omega)^{*} F(j \omega) \forall \omega \in \mathbb{R} \cup\{\infty\}$ [14].
} 
The following lemma derives a necessary and sufficient condition for a system to be OSNI and is a generalized version of [14, Lemma 6].

Lemma 2: (OSNI Lemma) Let $M(s) \in \mathscr{R} \mathscr{H}_{\infty}^{m \times m}$ have a minimal state-space realization $(A, B, C, D)$. Let $\delta>0$ be a scalar. Then, $M(s)$ is OSNI with a level of output strictness $\delta$ if and only if $D=D^{T}$ and there exists a real matrix $Y=Y^{T}>0$ such that

$A Y+Y A^{T}+\delta(C A Y)^{T} C A Y \leq 0$ and $B+A Y C^{T}=0$. (8)

Proof: For the sake of convenience, we denote a shorthand

$$
\Pi=\left[\begin{array}{cc}
\left(\begin{array}{c}
-P A-A^{T} P \\
-\delta A^{T} C^{T} C A
\end{array}\right) & \left(\begin{array}{c}
-P B+A^{T} C^{T} \\
-\delta A^{T} C^{T} C B
\end{array}\right) \\
\left(\begin{array}{cc}
-P B+A^{T} C^{T} \\
-\delta A^{T} C^{T} C B
\end{array}\right)^{T} & \left(\begin{array}{c}
C B+B^{T} C^{T} \\
-\delta B^{T} C^{T} C B
\end{array}\right)
\end{array}\right] .
$$

The proof proceeds through the following sequence of equivalent statements:

$M(s)$ is OSNI with a level of output strictness $\delta$ $\Leftrightarrow M(s)-D$ is OSNI with a level of output strictness $\delta$ and $D=D^{T}$ $\Leftrightarrow F(s)=s[M(s)-D]=(A, B, C A, C B)$ is OSP with a a level of output strictness $\delta$ and $D=D^{T}$ [via Lemma 1] $\Leftrightarrow D=D^{T}$ and there exists $P=P^{T}>0$ such that

$\Pi \geq 0$ [invoking [14, Lemma 2] with $\delta>0$ and $\varepsilon=0$ ] $\Leftrightarrow D=D^{T}$ and there exists $Y=Y^{T}>0$ such that

$A Y+Y A^{T}+\delta(C A Y)^{T} C A Y \leq 0$ and $B+A Y C^{T}=0$ [On letting $Y=P^{-1}$ and following the same algebraic manipulation as in the proof of [14, Lemma 5]].

This completes the proof.

Note that the matrix inequality in (8) is not in LMI form but can be readily converted into an LMI by applying the Schur Complement Lemma [25, Appendix A.61].

\section{CONNECTIONS BETWEEN OSNI SYSTEMS PROPERTY AND DISSIPATIVITY}

This section is a main contribution of this paper. Subsection IV-A extends the classical notion of dissipativity to include supply rates that involve time derivative of the system's output being inspired by [18], [21], [23] and introduces a time domain dissipative framework for characterising the OSNI as well as stable NI systems. While, subsection IV-B shows the equivalence between the state-space characterisation and time domain dissipativity of the class of OSNI systems.

\section{A. OSNI systems in time domain dissipative framework}

The following theorem establishes that for an initially relaxed OSNI system $M$ with a reachable (from origin) statespace, there always exists a positive semidefinite storage function $V(x)$ such that the system satisfies the dissipation inequality (2) with a particular time domain supply rate $w(u, \dot{\bar{y}})=2 \dot{\bar{y}}^{T} u-\delta \dot{\bar{y}}^{T} \dot{\bar{y}}$ with $\delta>0$, where $\bar{y}=y-D u$ is defined as an auxiliary output of $M$. Note that in this section, the admissible inputs $u(t)$ are taken to be $\mathbb{R}^{m}$-valued locally square integrable functions of time $t \in \mathbb{R}_{>0}$ along with sufficient smoothness properties such that $\dot{\bar{y}}(t)=C \dot{x}(t)=$ $C A x(t)+C B u(t)$ exists and belongs to $\mathbb{R}^{m}$ for all $t \in \mathbb{R}_{\geq 0}$, and also, $\dot{\bar{y}}(t)$ remains locally square integrable.

Theorem 1: Let $M$ be a causal, square, LTI system given by the state-space equations $\dot{x}=A x+B u$ and $y=C x+D u$ with zero initial condition and the state-space being reachable from the origin. Let the associated transfer function matrix be $M(s) \in \mathscr{R} \mathscr{H}_{\infty}^{m \times m}, \delta>0$ and $\bar{y}=y-D u$. Then, $M$ is dissipative with respect to the supply rate $w(u, \dot{\bar{y}})=$ $2 \dot{\bar{y}}^{T} u-\delta \dot{\bar{y}}^{T} \dot{\bar{y}}$ if $M(s)$ is OSNI with the same $\delta$.

Proof: To show that the OSNI system $M$ with $\delta>0$ is dissipative with respect to the supply rate $w(u, \dot{\bar{y}})=$ $2 \dot{\bar{y}}^{T} u-\delta \dot{\bar{y}}^{T} \dot{\bar{y}}$, we have to establish that there exists a storage function $V: \mathbb{R}^{n} \rightarrow \mathbb{R}_{\geq 0}$ such that $M$ satisfies the dissipation inequality (2). Since the state-space is assumed to be reachable from the origin, there exists an admissible input $u(t)$ defined as

$$
u(t)= \begin{cases}0 & \text { when } t<t_{-1} \\ \tilde{u}(t) & \text { when } t_{-1} \leq t \leq 0 \\ 0 & \text { when } t>0\end{cases}
$$

which steers the system from $x\left(t_{-1}\right)=0$ to any $x(0) \in$ $\mathbb{R}^{n}$. In this proof, let $y(t)$ be the corresponding output; $Y(j \omega), \bar{Y}(j \omega)$ and $U(j \omega)$ denote respectively the Fourier Transform of the real-valued time domain signals $y(t), \bar{y}(t)$ and $u(t) \cdot \bar{Y}(j \omega)=Y(j \omega)-D U(j \omega)=\bar{M}(j \omega) U(j \omega)$ where $\bar{M}(j \omega)=M(j \omega)-D$. Now,

$$
\begin{aligned}
& \int_{t_{-1}}^{0} w(u, \dot{\bar{y}}) \mathrm{d} t=\int_{t_{-1}}^{0}\left(2 \dot{\bar{y}}^{T} u-\delta \dot{\bar{y}}^{T} \dot{\bar{y}}\right) \mathrm{d} t \\
& =\int_{-\infty}^{\infty}\left(2 \dot{\bar{y}}^{T} u-\delta \dot{\bar{y}}^{T} \dot{\bar{y}}\right) \mathrm{d} t+\delta \int_{0}^{\infty} \dot{\bar{y}}^{T} \dot{\bar{y}} \mathrm{~d} t
\end{aligned}
$$

[since $M$ is causal and time-invariant]

$\geq \int_{-\infty}^{\infty}\left(2 \dot{\bar{y}}^{T} u-\delta \dot{\bar{y}}^{T} \dot{\bar{y}}\right) \mathrm{d} t \quad[$ since $\delta>0]$

$=\frac{1}{2 \pi} \int_{-\infty}^{\infty}\left[(j \omega \bar{Y}(j \omega))^{*} U(j \omega)+U(j \omega)^{*}(j \omega \bar{Y}(j \omega))-\right.$

$\left.\delta \omega^{2} \bar{Y}(j \omega)^{*} \bar{Y}(j \omega)\right] \mathrm{d} \omega$ [applying Parseval's identity [25]]

$$
\begin{array}{r}
=\frac{1}{2 \pi} \int_{-\infty}^{\infty} U(j \omega)^{*}\left[j \omega\left\{M(j \omega)-M(j \omega)^{*}\right\}-\right. \\
\left.\delta \omega^{2} \bar{M}(j \omega)^{*} \bar{M}(j \omega)\right] U(j \omega) \mathrm{d} \omega
\end{array}
$$

[since $M(\infty)=M(\infty)^{T}$ is implied by (7)]

\section{$\geq 0 \quad$ [using Definition 5 and Remark 1].}

Hence for arbitrary $t_{-1} \leq 0$ and $x\left(t_{-1}\right)=0$, we have $\int_{t_{-1}}^{0} w(u, \dot{\bar{y}}) \mathrm{d} t \geq 0$. We now construct the required supply function as $V_{r}(x)=\inf _{\substack{x^{*}=0 \rightarrow x \\ u(\cdot), t_{-1} \leq 0}} \int_{t_{-1}}^{0} w(u, \dot{\bar{y}}) \mathrm{d} t \geq 0$ where origin is the point of minimum storage (i.e., $x^{*}=0$ ). Thus, $V_{r}(x)$ can be considered as a storage function candidate associated with the OSNI system $M$.

It remains to be shown that $V_{r}(x)$ satisfies the dissipation inequality (2). Towards this end, note that in taking the 
system from $x=0$ at $t=0$ to $x_{1} \in \mathbb{R}^{n}$ at $t=t_{1}$, we could first take it to $x_{0} \in \mathbb{R}^{n}$ at time $t_{0}$ while minimizing the energy, and then take it to $x_{1}$ at time $t_{1}$ along the path for which the dissipation inequality is to be evaluated. This is possible by virtue of $M$ being a causal and timeinvariant system. Since $V_{r}\left(x_{1}\right)$ gives the infimum of the amount of energy required to reach $x_{1}$ at $t=t_{1}$ from $x=0$ at $t=0$, the energy required to reach the same destination $x_{1}$ from the same starting point $x=0$ via any other path will be greater than or equal to $V_{r}\left(x_{1}\right)$. Therefore, $V_{r}\left(x_{0}\right)+\int_{t_{0}}^{t_{1}} w(u, \dot{\bar{y}}) \mathrm{d} t \geq V_{r}\left(x_{1}\right)$ follows. It can hence be concluded that the OSNI system $M$ is dissipative with respect to the supply rate $w(u, \dot{\bar{y}})=2 \dot{\bar{y}}^{T} u-\delta \dot{\bar{y}}^{T} \dot{\bar{y}}$ for the same $\delta>0$.

Following Theorem 1, a similar dissipative characterisation can be given for all stable NI (i.e., with $\delta=0$ ) systems.

Lemma 3: Let $M(s) \in \mathscr{R} \mathscr{H}_{\infty}^{m \times m}$ be the transfer function matrix of an NI system $M$ with $\delta=0$ and it has a reachable state-space from the origin. Let $M$ have time domain input $u$, time domain output $y$ and define $\bar{y}=y-M(\infty) u$. Then, $M$ is dissipative with respect to the supply rate $w(u, \dot{\bar{y}})=2 \dot{\bar{y}}^{T} u$.

Proof. The proof readily follows from Theorem 1 by setting $\delta=0$ and applying the frequency domain criteria $j \omega\left[M(j \omega)-M(j \omega)^{*}\right] \geq 0$ for all $\omega \in \mathbb{R} \cup\{\infty\} \Leftrightarrow$ $j\left[M(j \omega)-M(j \omega)^{*}\right] \geq 0$ for all $\omega \in(0, \infty)$, since the latter implies $M(0)=M(0)^{T}$ and $M(\infty)=M(\infty)^{T}$ for all stable NI systems via Remark 1 .

The following example shows that an OSNI system satisfies the time domain dissipation inequality with the proposed supply rate $w(u, \dot{\bar{y}})=2 \dot{\bar{y}}^{T} u-\delta \dot{\bar{y}}^{T} \dot{\bar{y}}$, once an appropriate storage function is chosen.

Example 4: Consider the OSNI transfer function $M(s)=$ $\frac{25}{s^{2}+5 s+25}$ with a minimal state-space description $\dot{x_{1}}=$ $x_{2}, \dot{x_{2}}=-25 x_{1}-5 x_{2}+25 u$ and $y=x_{1}$ with $x(0)=0$. For this system, without loss of generality, we can choose a positive definite storage function $V(x)=x_{1}^{2}+\frac{1}{25} x_{2}^{2}$ such that $w(u, \dot{\bar{y}})-\dot{V}(x)=\left(2 \dot{\bar{y}}^{T} u-\delta \dot{\bar{y}}^{T} \dot{\bar{y}}\right)-\left[\frac{\partial V(x)}{\partial x}\right]^{T} \dot{x}=$ $(0.4-\delta) x_{2}^{2} \geq 0$ for all $\delta \in[0,0.4]$ and all admissible inputs $u(t) \in \mathbb{R}$ for all $t \in \mathbb{R}_{\geq 0}$. Therefore, $M(s)$ is dissipative with respect to the time domain supply rate $w(u, \dot{\bar{y}})$ with $\delta \in[0,0.4]$. Note, $\delta=0$ indicates that $M(s)$ is also a stable NI system (see Remark 1).

\section{B. Equivalence between time domain dissipativity and state- space characterisation of OSNI systems}

We have already established that OSNI systems (with $\delta>$ $0)$ are dissipative with respect to the time domain supply rate $w(u, \dot{\bar{y}})=2 \dot{\bar{y}}^{T} u-\delta \dot{\bar{y}}^{T} \dot{\bar{y}}$ where $\bar{y}=y-D u$ is selected as an auxiliary output of the system. In this subsection, we will show that for a stable LTI system with a minimal statespace realisation, the OSNI Lemma conditions are equivalent to time domain dissipativity with respect to the proposed supply rate $w(u, \dot{\bar{y}})$ and a specific storage function given by $V(x)=x^{T} P x$ with $P=P^{T}>0$ for all $x \in \mathbb{R}^{n}$.
Theorem 2: Let $M$ be a causal, square, LTI system described by the state-space equations $\dot{x}=A x+B u, x(0)=0$ and $y=C x+D u$, where $A$ is Hurwitz, $D=D^{T}$ and $(A, B, C, D)$ is minimal. Let the associated transfer function matrix be $M(s)$ and define $\bar{y}=y-D u$. Let a scalar $\delta>0$. Then, the following statements are equivalent:

i) $M(s)$ is OSNI with a level of output strictness $\delta$;

ii) there exists a real matrix $Y=Y^{T}>0$ such that

$$
A Y+Y A^{T}+\delta(C A Y)^{T}(C A Y) \leq 0 \text { and } B=-A Y C^{T} ;
$$

iii) $M$ is dissipative with respect to the supply rate $w(u, \dot{\bar{y}})=2 \dot{\bar{y}}^{T} u-\delta \dot{\bar{y}}^{T} \dot{\bar{y}}$.

Proof: $i) \Leftrightarrow i i)$ This equivalence is due to OSNI Lemma. ii $) \Rightarrow$ iii) There exists a real matrix $Y=Y^{T}>0$ such that

$$
A Y+Y A^{T}+\delta(C A Y)^{T}(C A Y) \leq 0 \text { and } B=-A Y C^{T}
$$
$\Leftrightarrow$ there exists $P=P^{T}>0$ such that $\Pi \geq 0 \quad$ [on letting $P=Y^{-1}$ and following the proof of Lemma 2. The shorthand $\Pi$ has been introduced in (9)] $\Leftrightarrow$ there exists $P=P^{T}>0$ such that

$$
\left[\begin{array}{ll}
x^{T} & u^{T}
\end{array}\right] \Pi\left[\begin{array}{l}
x \\
u
\end{array}\right] \geq 0 \text { for all }\left[\begin{array}{l}
x \\
u
\end{array}\right] \in \mathbb{R}^{n+m}
$$

$\Leftrightarrow$ there exists $P=P^{T}>0$ such that $2(C A x+C B u)^{T} u$

$-\delta(C A x+C B u)^{T}(C A x+C B u) \geq x^{T}\left(P A+A^{T} P\right) x$

$+2 x^{T} P B u$ for all $x \in \mathbb{R}^{n}$ and $u \in \mathbb{R}^{m}$

$\Rightarrow$ there exists $P=P^{T}>0$ such that the differentiable storage function $V(x)=x^{T} P x$ satisfies $2 \dot{\bar{y}}^{T} u-\delta \dot{\bar{y}}^{T} \dot{\bar{y}}$ $\geq \dot{V}(x)$ evaluated along any trajectory of $M$ subjected to any admissible input $u(t) \in \mathbb{R}^{m}$ for all $t \in \mathbb{R}_{\geq 0}$

[Note $x, y, \bar{y}$ all are now time variables, that is, $x=x(t)$ $\in \mathbb{R}^{n}, y=y(t) \in \mathbb{R}^{m}, \bar{y}=\bar{y}(t) \in \mathbb{R}^{m}$ for all $\left.t \in \mathbb{R}_{\geq 0}\right]$ $\Leftrightarrow$ there exists a differentiable storage function $V(x)=$ $x^{T} P x$ with $P=P^{T}>0$ such that $\int_{0}^{T} 2 \dot{\bar{y}}^{T} u-\delta \dot{\bar{y}}^{T} \dot{\bar{y}} \mathrm{~d} t$ $\geq V(x(T))-V(x(0))$ for all $T \in[0, \infty)$ and evaluated along any trajectory of $M$ subjected to any admissible input $u(t) \in \mathbb{R}^{m}$ for all $t \in \mathbb{R}_{\geq 0}$

$\Leftrightarrow M$ is dissipative with respect to the supply rate $w(u, \dot{\bar{y}})$

$=2 \dot{\bar{y}}^{T} u-\delta \dot{\bar{y}}^{T} \dot{\bar{y}}$ and a specific storage function $V(x)=$ $=x^{T} P x$ with $P=P^{T}>0$.

$i i i) \Rightarrow i i)$ This follows via the necessity part of the proof of [17, Theorem 1]. Suppose there exists a differentiable storage function $V(x)=x^{T} P x$ with $P=P^{T}>0$ such that

$$
2 \dot{\bar{y}}^{T} u-\delta \dot{\bar{y}}^{T} \dot{\bar{y}} \geq \dot{V}(x)
$$

along any trajectory of $M$ for any admissible input $u(t) \in$ $\mathbb{R}^{m}$ for all $t \in \mathbb{R}_{\geq 0}$. To turn this inequality into equality, let us introduce a function $d: \mathbb{R}^{n} \times \mathbb{R}^{m} \rightarrow \mathbb{R}$ so that

$$
2 \dot{\bar{y}}^{T} u-\delta \dot{\bar{y}}^{T} \dot{\bar{y}}=\dot{V}(x)+d(\cdot, \cdot) .
$$


Note that $x, y, \bar{y}$ and $u$ all are now variables of time and they take real vectored values at each $t \in \mathbb{R}_{\geq 0}$. As

$$
\left(2 \dot{\bar{y}}^{T} u-\delta \dot{\bar{y}}^{T} \dot{\bar{y}}\right)-\dot{V}(x)=\left[\begin{array}{ll}
x^{T} & u^{T}
\end{array}\right] \Pi\left[\begin{array}{l}
x \\
u
\end{array}\right],
$$

it is evident that $d(\cdot, \cdot)$ is a function of both $x$ and $u$, and $d(\cdot, \cdot)$ must be quadratic in $x$ and $u$. Moreover, $d(\cdot, \cdot) \geq 0$ is implied from (10). Based on these observations, $d(x, u)$ can be factored as

$$
d(x, u)=(L x+W u)^{T}(L x+W u)
$$

for some suitable choice of the matrices $L \in \mathbb{R}^{m \times n}$ and $W \in \mathbb{R}^{m \times m}$. Note, the choice of $L$ and $W$ may not be unique. Substituting (12) and (13) into (11), we have

$$
\begin{aligned}
{\left[\begin{array}{ll}
x^{T} & u^{T}
\end{array}\right] \Pi\left[\begin{array}{l}
x \\
u
\end{array}\right]=} & {\left[\begin{array}{cc}
x^{T} & u^{T}
\end{array}\right] } \\
& {\left[\begin{array}{cc}
L^{T} L & L^{T} W \\
W^{T} L & W^{T} W
\end{array}\right]\left[\begin{array}{l}
x \\
u
\end{array}\right] . }
\end{aligned}
$$

Since $L$ and $W$ are constant matrices, (14) holds for all $x \in \mathbb{R}^{n}$ and all $u \in \mathbb{R}^{m}$, not necessarily related by the state equations. Equating the coefficients of the terms involving $x$ and $u$, we obtain the set of equality conditions

$$
\begin{aligned}
& -P A-A^{T} P-\delta A^{T} C^{T} C A=L^{T} L, \\
& -P B+A^{T} C^{T}-\delta A^{T} C^{T} C B=L^{T} W, \quad \text { and } \\
& C B+B^{T} C^{T}-\delta B^{T} C^{T} C B=W^{T} W,
\end{aligned}
$$

which is equivalent to the set of conditions $A Y+Y A^{T}+$ $\delta(C A Y)^{T}(C A Y) \leq 0$ and $B+A Y C^{T}=0$. This completes the proof.

\section{CONCLUSIONS}

This paper generalises the notion of output strictly negative imaginary systems (OSNI) introduced in [14] to widen the applicability of this theory to more general class of LTI systems. The present work has also underpinned the connections between negative imaginary (NI) theory and classical dissipativity. It is established that the class of OSNI systems is equivalent to a class of dissipative systems with respect to a particular supply rate comprised of the time domain input $(u)$, the time derivative of an auxiliary output of the system $(\dot{\bar{y}})$ where $\bar{y}$ is defined as $y-D u$ and a real parameter $\delta>0$. This dissipative characterisation may be extended in future to develop a unified framework for analysis and synthesis of NI and SNI systems.

\section{REFERENCES}

[1] A. Lanzon and I. R. Petersen, "Stability robustness of a feedback interconnection of systems with negative imaginary frequency response," IEEE Transactions on Automatic Control, vol. 53, no. 4, pp. 10421046, 2008.

[2] J. Xiong, I. R. Petersen and A. Lanzon, "A negative imaginary lemma and the stability of interconnections of linear negative imaginary systems," IEEE Transactions on Automatic Control, vol. 55, no. 10, pp. 2342-2347, 2010.

[3] A. Lanzon, Z. Song, S. Patra and I. R. Petersen, "A strongly strict negative imaginary lemma for non-minimal linear systems," Соттиnications in Information and Systems, vol. 11, no. 2, pp. 139-152, 2011.
[4] B. Bhikkaji, S. O. R. Moheimani and I. R. Petersen, "A negative imaginary approach to modeling and control of a colocated structure," IEEE/ASME Transactions on Mechatronics, vol. 17, no. 4, pp. 717727, 2012.

[5] C. Cai and G. Hagen, "Stability analysis for a string of couple stable subsystems with negative imaginary frequency response," IEEE Transactions on Automatic Control, vol. 55, no. 8, pp. 1958-1963, 2010.

[6] J. L. Fanson and T. K. Caughey, "Positive position feedback control for large space structures," AIAA Journal, vol. 28, no. 4, pp. 717-724, 1990.

[7] Z. Song, A. Lanzon, S. Patra and I. R. Petersen, "Robust performance analysis for uncertain negative imaginary systems," International Journal of Robust and Nonlinear Control, vol. 22, no. 3, pp. 262-281, 2012.

[8] S. Patra and A. Lanzon, "Stability analysis of interconnected systems with 'mixed' negative imaginary and small-gain properties," IEEE Transactions on Automatic Control, vol. 56, no. 6, pp. 1395-1400, 2011.

[9] S. K. Das, H. R. Pota and I. R. Petersen, "Stability analysis for interconnected systems with 'mixed' negative imaginary and passivity," in Proceedings of Australian Control Conference, Perth, Australia, 2013.

[10] M. A. Mabrok, A. G. Kallapur, I. R. Petersen and A. Lanzon, "Generalizing negative imaginary systems theory to include free body dynamics: Control of highly resonant structures with free body motion," IEEE Transactions on Automatic Control, vol. 59, no. 10, pp. 2692-2707, 2014.

[11] M. A. Mabrok, A. G. Kallapur, I. R. Petersen and A. Lanzon, "Spectral conditions for negative imaginary systems with applications to nanopositioning," IEEE/ASME Transactions on Mechatronics, vol. 19, no. 3, pp. 895-903, 2014.

[12] S. K. Das, H. R. Pota and I. R. Petersen, "Damping Controller Design for Nanopositioners: A Mixed Passivity, Negative-Imaginary and Small-Gain Approach," IEEE/ASME Transactions on Mechatronics, vol. 20, no. 1, pp. 416-426, 2015.

[13] A. Lanzon and H. -J. Chen, "Feedback stability of negative imaginary systems," IEEE Transactions on Automatic Control, vol. 62, no. 11, pp. 5620-5633, 2017.

[14] P. Bhowmick and S. Patra, "On LTI output strictly negative-imaginary systems," Systems \& Control Letters, vol. 100, pp. 32-42, 2017.

[15] P. Bhowmick and S. Patra, "An observer-based control scheme using negative-imaginary theory," Automatica, vol. 81, pp. 196-202, July, 2017.

[16] J. C. Willems, "Dissipative dynamical systems- Part I: General Theory; Part II: Linear systems with quadratic supply rates," Archive for Rational Mechanics and Analysis, vol. 45, no. 5, pp. 321-393, 1972.

[17] P. J. Moylan and D. J. Hill, "The stability of nonlinear dissipative systems," IEEE Transactions on Automatic Control, vol. AC-21, no. 5, pp. 708-711, 1976.

[18] D. J. Hill and P. J. Moylan, "Stability results for nonlinear feedback systems," Automatica, vol. 13, no. 4, pp. 377-382, 1977.

[19] D. J. Hill and P. J. Moylan, "Dissipative dynamical system: basic input-output and state properties," Journal of The Franklin Institute, vol. 309, no. 5, pp. 327-357, 1980.

[20] T. Kailath, "Linear Systems," Prentice-Hall, Inc., Englewood Cliffs, New Jersey, USA, 1980.

[21] K. A. Morris and J. N. Juang, "Dissipative controller designs for second-order dynamic systems," IEEE Transactions on Automatic Control, vol. 39, no. 5, pp. 1056-1063, 1994.

[22] H. K. Khalil, "Nonlinear Systems," 3rd Edition, Prentice-Hall, Inc., Upper Saddle River, New Jersey, USA, 2002.

[23] D. Angeli, "Systems with counterclockwise input-output dynamics," IEEE Transactions on Automatic Control, vol. 51, no. 7, pp. 1130$1143,2006$.

[24] W. M. Griggs, B. D. O. Anderson and A. Lanzon, "A 'mixed' small gain and passivity theorem in the frequency domain," Systems \& Control Letters, vol. 56, pp. 596-602, 2007.

[25] B. Brogliato, R. Lozano, B. Maschke and O. Egeland, "Dissipative systems analysis and control: Theory and applications," 2nd Edition, Upper Saddle River, Springer-Verlag, New York, USA, 2007.

[26] N. Kottenstette, M. J. Mccourt, M. Xia, V. Gupta and P. J. Antsaklis, "On relationships among passivity, positive realness and dissipativity in linear systems," Automatica, vol. 50, no. 4, pp. 1003-1016, 2014. 